\title{
El proceso de acreditación de Especialistas en Pediatría en Chile
}

\author{
J.M. López
}

\begin{abstract}
Accreditation process of pediatric healthcare professionals in Chile

In Chile since 1984, the Autonomous Corporation for Certification of Medical Specialties (CONACEM) has certified 12,294 medical specialists. The Pediatrics discipline began its certification processes in 1984; it had certified 1,329 specialists by December, 2012. There are three possible ways to get certified: a) to have passed the Training Graduate Degree Program, taken by $57 \%$ of the applicants; b) to meet the requirements for Training in Practice for 5 years, achieved by $39 \%$ of applicants; c) to have been trained oversees according to validated programs. There are 11 accredited 3-year long university programs, which take place in Santiago and Provinces with a total annual capacity of 96 students. With the exception of doctors approved by accredited medical university programs, the rest of the applicants must pass a practical examination. A written examination has been added since 2002, whose approval is required to take the practical examination, which has proven to have good discrimination ( $22 \%$ average rejection in 11 years). The certifications granted today are good for 10 years and recertification is good for 7 years. The expiration of the certification process is related to recertification. This review discusses the reasons behind it and discusses the requirements and a table of credits to complete. Current legislation requires the action of at least one certification body, a condition that has been legally and uniquely granted by the Ministry of Health to CONACEM since 2/11/2014.
\end{abstract}

(Key words: Pediatric, Healthcare, certification, acreditation, CONACEM).

Rev Chil Pediatr 2014; 85 (5): 599-607

\section{RESUMEN}

Desde 1984 la Corporación Autónoma de Certificación de Especialidades Médicas (CONACEM) ha certificado 12.294 especialistas médicos en Chile. La disciplina de Pediatría inició su certificación en 1984, habiendo certificado hasta Diciembre 2012 a 1.329 especialistas. Para la certificación existen tres vías posibles: a) haber aprobado un Programa de Formación Universitario Acreditado, lo que hizo el 57\% de los postulantes; b) cumplir los requisitos de Adiestramiento en Práctica durante 5 años, como sucedió con el $39 \%$ de los postulantes; c) haber sido formado en el extranjero de acuerdo a programas convalidados. Existen 11 programas universitarios acreditados, de 3 años de duración, con un cupo total anual de 96 alumnos que se desarrollan en Santiago y Provincias. Salvo los médicos aprobados en los Programas Universitarios acreditados, el resto de los postulantes aprobar un examen práctico; desde 2002 se ha agregado un examen escrito, cuya aprobación es requisito para dar el práctico, y que ha resultado tener buena discriminación ( $22 \%$ de rechazo promedio en el

Recibido el 17 de julio de 2014, última versión aceptada el 17 de septiembre de 2014.

José M. López Moreno

Presidente de la Corporación Nacional Autónoma de Certificación de Especialidades Médicas (CONACEM).

E-mail: jmlopez@med.puc.cl conacem@entelchile.net 
período de 11 años). Las certificaciones dadas hoy, tienen una duración de 10 años al inicio y serán de 7 años en la recertificación. La caducidad de las certificaciones está aparejada al proceso de recertificación, de lo cual se discuten las razones que lo avalan; se presentan los requisitos y una tabla de créditos a cumplir, elaboradas por CONACEM. La legislación vigente y en proceso, requiere la actuación de al menos una entidad certificadora, condición que ha sido otorgada legalmente en forma única por el Ministerio de Salud a CONACEM a partir del 11/02/2014.

(Palabras clave: Pediatría, certificación, acreditación, CONACEM).

Rev Chil Pediatr 2014; 85 (5): 599-607

La certificación y recertificación de especialistas médicos es tema de gran actualidad. En este campo, la Corporación Nacional Autónoma de Certificación de Especialidades Médicas (CONACEM) tiene un papel preponderante. Hasta 1984, en Chile sólo existía el reconocimiento de especialista por las Universidades con programas de formación acreditados por la Asociación de Facultades de Medicina de Chile (ASOFAMECH). Luego esta responsabilidad quedó radicada en CONACEM, corporación de derecho privado, fundada en 1984 y constituida por representantes de la Academia de Medicina del Instituto de Chile, Asociación de Facultades de Medicina de Chile, Colegio Médico de Chile A.G. y las Sociedades Científicas, integrando el directorio de la Corporación en proporción de 4 delegados cada cual, salvo la Academia de Medicina que tiene uno. El Ministerio de Salud aporta un representante con derecho a voz y existe un Secretario. Junto al Directorio trabajan 54 Comités de Especialidades con 7 miembros cada uno, con dos representantes de ASOFAMECH, Colegio Médico de Chile A.G. y de la Sociedad Científica pertinente. El Presidente es nominado por el Directorio. A este total de 377 médicos se agregan los profesores examinadores, lo que significa que más de 500 médicos aportan su mejor saber, con mística encomiable, y sin remuneración alguna. Lo referente a CONACEM puede ser consultado en la página web (www.conacem.cl).

CONACEM posee características tales que han permitido su reconocimiento legal como única entidad certificadora de especialidades médicas a partir de febrero de 2014. Ellas son:
1) Representatividad, ya que su constitución incorpora los estamentos más prestigiados de la Medicina chilena en: academia, ciencia, asistencia y aspectos gremiales; 2) Experiencia probada en 29 años de trayectoria; 3) Reconocimiento. CONACEM ha certificado hasta diciembre de 2012 a 12.294 especialistas que voluntariamente lo han solicitado, lo que expresa el prestigio que goza ante los prestadores de salud, individuales o institucionales; 4) Mística. El hecho que 500 médicos trabajen por CONACEM, sólo por la satisfacción moral de apoyar a la medicina chilena y a los pacientes, es un galardón difícil de emular; 5) Transparencia. Los datos del proceso de certificación son obtenibles electrónicamente, $\mathrm{y}$ también comunicados a la Intendencia de Prestadores Médicos; 6) Independencia, desarrollando su labor ajena a consideraciones políticas, religiosas u otras.

CONACEM está centrada en la certificación de especialistas médicos y en la definición de especialidades médicas. No corresponde a ella la certificación de los programas de formación para especialistas, lo que es legalmente responsabilidad de agencias certificadoras, donde destaca la Agencia de Acreditación de Programas y Centros formadores de Especialistas (APICE).

\section{¿Qué define a una especialidad y a un especialista médico?}

Especialidad es un campo del saber médico que comprende armónicamente el conocimiento, las patologías y sus respectivos tratamientos. Especialista es aquel médico que domina el conocimiento, diagnóstico y tratamiento de 
las enfermedades de una disciplina reconocida como especialidad. En esta definición el vocablo "domina" marca el sentido de lo que es un especialista, concepto que repite el Decreto Afecto $\mathrm{N}^{\circ} 8$ publicado el 01/07/2013 en su Artículo $1^{\circ} \mathrm{b}$. La condición de especialista se abre también al campo de la investigación, docencia y divulgación científica.

\section{¿Qué tipos de especialidades se reconocen?}

Existen dos tipos de especialidades: a) Primarias, a cuya formación se puede acceder desde el inicio de la vida profesional, con el único requisito de ser médico cirujano. CONACEM certifica 26 especialidades primarias, entre ellas Pediatría; b) Derivadas que requieren de la certificación previa como especialista en la disciplina primaria de la cual se originan. CONACEM reconoce 31 especialidades derivadas, de las cuales 7 tienen a Pediatría como la especialidad primaria.

E1 Decreto Afecto $\mathrm{N}^{\circ} 8$, que entrará en vigencia el 01/01/2015, lista 52 especialidades médicas (Artículo 2), y, aunque diferencia la existencia de especialidades primarias y derivadas (Artículo 1b) no las individualiza en su condición; además, encarga al MINSAL elaborar las normas técnicas operativas de cada especialidad (Artículo 2) y crea las normas para incorporar nuevas especialidades (Artículo 13 ).

Una categoría especial es la Mención, que no constituye una especialidad, pero sí reconoce y avala la dedicación y capacitación en un área de una especialidad.

\section{¿Cuáles son las vías para certificarse como especialista en CONACEM?}

Existen 3 opciones: 1) Haber aprobado un Programa Universitario de formación acreditado por ASOFAMECH anteriormente, o APICE actualmente; 2) Por Formación en el Extranjero. Esta vía requiere que el Comité de la especialidad correspondiente haga un análisis de homologación entre el programa cursado en el extranjero, y los aprobados y vigentes en Chile. Si hay concordancia el candidato deberá someterse a un examen teórico y, de ser este aprobado, a uno práctico; 3 ) Por Adiestramiento en Práctica. Como requisito general el pos- tulante debe haber trabajado en la especialidad por mínimo 5 años, en jornada de al menos 22 $\mathrm{h}$ semanales, y con ligazón legal en centros de complejidad acorde con la disciplina, los cuales deben contar al menos con dos especialistas certificados que avalen el aprendizaje del candidato. Cada especialidad tiene requisitos adicionales específicos. Los candidatos deben rendir ante CONACEM un examen teórico $\mathrm{y}$, una vez aprobado éste, otro práctico.

Para definir una especialidad o subespecialidad CONACEM lo hace a requerimiento de algunos de sus socios (Academia de Medicina de Chile, Sociedades Científicas, ASOFA$\mathrm{MECH}$, Colegio Médico de Chile A.G.) o de los miembros del Directorio. Los requisitos básicos para iniciar ese estudio son: a) que exista legalmente una sociedad científica activa dedicada al área en análisis; b) que exista un programa universitario de formación acreditado en esa disciplina; c) que se cuente con una masa crítica mínima de 20 médicos ejerciendo la especialidad; d) que la disciplina a reconocer sea concordante con la tradición y los valores de la medicina chilena.

\section{¿Cuál es la situación legal del reconocimiento de especialistas?}

Hasta 1984 el reconocimiento lo hacían las universidades con programas acreditados de formación. En 1984 CONACEM inicia la certificación de especialistas. En Febrero del 2004 se dictó la ley 19.937 de Autoridad Sanitaria que establece un sistema de certificación de especialistas y encomienda a los Ministerios de Salud y Educación confeccionar un reglamento para determinar las entidades examinadoras y las condiciones para que ellas sean autorizadas. El 6 de noviembre de 2008 se dicta el Decreto Supremo N ${ }^{\circ} 57$ que responde a esa ordenanza y define 24 especialidades médicas y 18 subespecialidades, fija los requisitos y procedimientos, señala las modalidades para otorgar la certificación, y define la vigencia de la certificación por un período entre 5 y 10 años. Además, crea un registro público de prestadores individuales. En atención a que el proceso organizativo sufría retraso un nuevo Decreto Supremo, dictado el 2010, prorrogó hasta el año 2012 ciertos aspectos transitorios 
del Decreto Supremo N ${ }^{\circ} 57$ y que tenían relación con las entidades certificadoras, las que podrían registrarse e iniciar sus actividades en ese tiempo de prórroga. Además, reconocía que las certificaciones vigentes a esa fecha (2008) serían válidas por un plazo de 7 años. En esta prórroga incluía las certificaciones como especialistas otorgadas por las universidades y por CONACEM, los reconocimientos de desempeño en práctica de la especialidad y los médicos listados en los convenios de especialidad con FONASA. Posteriormente, el 27/06/2013 la Contraloría General de la República tomó razón del decreto $\mathrm{N}^{\mathrm{o}} 8$ sobre "Reglamento de certificación de las especialidades de los prestadores individuales de salud y de las entidades que los otorgan". Este reglamento señala un listado de 52 especialidades, define que corresponde al MINSAL dictar las normas técnicas operativas que permitan uniformar y homologar los mecanismos y procedimientos que deberán dar a conocer y exigir las entidades certificadoras autorizadas, describe el modo como el MINSAL verificará el cumplimiento de los requisitos para incorporar nuevas especialidades, y la validez temporal de las certificaciones (ver más adelante).

Este reglamento entrará en vigencia el 01/01/2015, y a partir de su publicación en el Diario Oficial se deroga el decreto Supremo $\mathrm{N}^{\mathrm{o}} 57$ del 14/02/2007.

El 11/02/2014 el Ministerio de Salud reconoce legalmente a CONACEM como la única entidad certificadora de prestadores individuales de 24 especialidades médicas, las que serían ampliadas en un futuro cercano hasta llegar al total de ellas. Anestesiología integra ya el listado inicial.

\section{¿Qué es certificar y recertificar?}

Según la Real Academia de la Lengua Española certificar es asegurar, afirmar y dar por cierto algo, en este caso la calidad de especialista. También es hacer constar por escrito una realidad de hecho por quien tenga fe pública o atribución para ello. No se aparta de esta definición la que hace el Decreto Supremo $N^{\circ} 57$ que señala que es un "proceso en virtud del cual se reconoce que un prestador individual domina un cuerpo de conocimientos y/o expe- riencias relevantes en un determinado ámbito del trabajo asistencial, para el otorgamiento del correspondiente certificado. Recertificar es refrendar la certificación de modo que las constataciones hechas inicialmente mantengan valor y actualidad.

La recertificación requiere de una certificación previa y por lo tanto aquellos médicos incluidos en categorías reconocidas por la ley (constancia de desempeño de la especialidad, convenio con FONASA), deben, como primera instancia, certificarse.

Esta definición de especialista, corre el riesgo potencial de ser banalizada. Minimizar o alivianar lo que es un verdadero especialista, muchas veces por la urgencia de la contingencia, implica un menoscabo a la medicina porque: a) rompe con la fe pública en los organismos de la sociedad encargados de certificar que lo que se dice es verdadero; b) soluciona parcialmente los problemas que se pretenden abordar; c) disminuye la esperada eficacia y eficiencia del especialista; d) aumenta los costos en tiempo y dinero; e) genera inequidad para con los médicos que tienen la calidad de especialistas respecto de aquellos que no la cumplen.

La definición laxa de lo que es una especialidad o subespecialidad tiene el riesgo de la fragmentación exagerada del cuerpo de conocimientos de la especialidad. La mayor tendencia a que ello ocurra sucede cuando se desarrollan actividades médicas centradas en una tecnología de compleja operatividad.

\section{¿Qué vigencia tienen las certificaciones?}

Las universidades, cuando otorgaban el título de especialista, lo consideraron vitalicio. Para CONACEM las certificaciones previas a 2008 eran también permanentes; después, en atención a la nueva legislación se extendieron por 10 años en primera instancia, para que las renovaciones lo fueran por 7 años. A partir de agosto de 2008 y hasta diciembre de 2012 CONACEM ha certificado 97 especialistas pediatras, con vigencia de 10 años.

En el período comprendido entre la publicación en el Diario Oficial del Decreto Afecto $\mathrm{N}^{\circ} 8$ y su entrada en vigencia $(01 / 01 / 2015)$ las entidades certificadoras pueden constituir- 
se, registrarse e iniciar sus procedimientos de certificación. Los artículos transitorios del DS $\mathrm{N}^{\circ} 57$ anterior se mantienen. Así, se reconocerán como certificados los profesionales que entre el 01/01/2013 y 31/12/2014 se encuentren en alguna de las siguientes situaciones: a) Que obtengan el título de especialista por una universidad del estado o reconocida por este y cuya vigencia será de 10 años a contar de la obtención del título (Artículo Transitorio 1); b) Que hayan sido certificados por CONACEM y se encuentren desempeñando actividades propias de la especialidad a la fecha de la publicación de este Reglamento; la vigencia será de 7 años desde la obtención del título (Artículo Transitorio 2); c) Los que detenten constancia de desempeño en la práctica en establecimientos del SNSS, de la Defensa Nacional, Carabineros de Chile o de su Dirección de Previsión o en el Servicio Médico Legal, por un período no menor a 5 años. La certificación será reconocida por 7 años desde el momento de su otorgamiento (Artículo Transitorio 3). Los reconocimientos de certificación de especialidades otorgados según el Decreto $\mathrm{N}^{\circ} 57$ del 2007 del MINSAL, mantendrán su vigencia por el mismo plazo en que fueron otorgados. Los profesionales con certificación de especialidad por tener un título o grado Académico en esa especialidad, otorgado por una Universidad del Estado, o reconocido por este, la vigencia del reconocimiento expirará el $06 / 11 / 2018$.

\section{¿Qué razones avalan la recertificación?}

Son variadas: a) complejidad creciente de los conocimientos, tecnologías, destrezas, y fuentes de información, con emergencia de nuevos campos del saber y del actuar médico, que deben acotarse; b) rápida obsolescencia del conocimiento médico y de sus paradigmas; c) divulgación amplia e instantánea de los avances mundiales en medicina; d) demanda de los usuarios por contar con medicina del más alto nivel y efectividad; e) necesidad de asegurar la calidad de las prestaciones médicas por parte de las instituciones prestadoras de salud, y de los propios médicos; f) creciente judicialización de las acciones médicas que requiere clarificar responsabilidades y responder al concepto jurídico de "temeridad o no temeridad médica”; g) cumplir con la Ley N 19.966 del Régimen General de Garantías en Salud, referentes al acceso, calidad y oportunidad de las acciones médicas.

\section{¿Qué ha sucedido con la Pediatría mirada desde CONACEM?}

El proceso de certificación en Pediatría se inició en 1984 y desde entonces un total de 1589 médicos han solicitado su certificación, lográndolo 1.329 postulantes $(83,6 \%)$. Pediatría, como especialidad primaria, es requisito para la certificación de 7 subespecialidades: Cardiología, Endocrinología Pediátrica, Enfermedades Respiratorias Pediátricas, Gastroenterología Pediátrica, Hemato-Oncología Pediátrica, Medicina Intensiva Pediátrica y Nefrología Pediátrica.

Inicialmente, se reconoció como especialistas a los Profesores universitarios de la disciplina (118 en total), modalidad que fue eliminada el 2004. El 93,2\% de los médicos que la utilizó lo hizo antes de 1999. El universo restante de 1211 especialistas corresponde mayoritariamente $(57 \%)$ a Programas Universitarios acreditados.

A la fecha, están acreditados 11 programas de formación, de 3 años de duración. El cupo global de alumnos del conjunto es de 96 vacantes por año. Del total de 11 programas 6 se desarrollan en Santiago, y el resto en provincias (Antofagasta, Valparaíso, Concepción, Temuco y Valdivia). La distribución geográfica de los alumnos señala que $61 \%$ de ellos son de Santiago y el resto de provincias.

La vía de Adiestramiento en Práctica ha sido usada por el $39 \%$ de los especialistas y la de Formación en el Extranjero es mínima (4\%) no sobrepasando en promedio 2 médicos por año.

El desglose quinquenal de las diferentes vías se presenta en la Tabla 1. El primer quinquenio se aleja de la tónica general dado que corresponde al inicio del proceso de certificación y que incluye a los Profesores Universitarios.

A partir de 1990, el promedio anual de postulaciones y certificaciones ha aumentado llegando a duplicarse; el tramo 2009-2012, 
Tabla 1. Número, promedio anual y distribución porcentual de las certificaciones de especialistas en Pediatría, distribuidos por quinquenios, según, la vía usada

\begin{tabular}{|c|c|c|c|c|c|c|c|}
\hline Período & & PU & AP & FE & Subtotal & Prof. Univ. & Total \\
\hline $1984-1988$ & $\begin{array}{l}\mathrm{n} \\
\overline{\mathrm{x}} \text { año } \\
\%\end{array}$ & $\begin{array}{r}193,0 \\
38,6 \\
35,3\end{array}$ & $\begin{array}{r}280,0 \\
56,0 \\
61,3\end{array}$ & $\begin{array}{r}11,0 \\
2,2 \\
2,4\end{array}$ & 484,0 & 85 & 569 \\
\hline $1989-1993$ & $\begin{array}{l}\mathrm{n} \\
\overline{\mathbf{x}} \text { año } \\
\%\end{array}$ & $\begin{array}{l}66,0 \\
13,2 \\
60,0\end{array}$ & $\begin{array}{r}40,0 \\
8,0 \\
36,4\end{array}$ & $\begin{array}{l}4,0 \\
0,8 \\
3,6\end{array}$ & 110,0 & 11 & 121 \\
\hline $1994-1998$ & $\begin{array}{l}\mathrm{n} \\
\overline{\mathbf{x}} \text { año } \\
\%\end{array}$ & $\begin{array}{r}48,0 \\
9,6 \\
47,1\end{array}$ & $\begin{array}{r}46,0 \\
9,2 \\
45,1\end{array}$ & $\begin{array}{l}8,0 \\
1,6 \\
7,8\end{array}$ & 102,0 & 14 & 116 \\
\hline $1999-2003$ & $\begin{array}{l}\mathrm{n} \\
\overline{\mathbf{x}} \text { año } \\
\%\end{array}$ & $\begin{array}{r}132,0 \\
26,4 \\
73,7\end{array}$ & $\begin{array}{r}41,0 \\
8,2 \\
22,9\end{array}$ & $\begin{array}{l}6,0 \\
1,2 \\
3,4\end{array}$ & 179,0 & 6 & 185 \\
\hline $2004-2008$ & $\begin{array}{l}\mathrm{n} \\
\overline{\mathrm{x}} \text { año } \\
\%\end{array}$ & $\begin{array}{r}185,0 \\
37,0 \\
78,7\end{array}$ & $\begin{array}{r}40,0 \\
8,0 \\
17,0\end{array}$ & $\begin{array}{r}10,0 \\
2,0 \\
4,3\end{array}$ & 235,0 & 2 & 237 \\
\hline $2009-2012$ & $\begin{array}{l}\mathrm{n} \\
\bar{x} \text { año } \\
\%\end{array}$ & $\begin{array}{l}69,0 \\
17,3 \\
68,3\end{array}$ & $\begin{array}{r}23,0 \\
5,8 \\
22,8\end{array}$ & $\begin{array}{l}9,0 \\
2,3 \\
8,9\end{array}$ & 101,0 & & 101 \\
\hline Totales & $\begin{array}{l}\mathrm{n} \\
\%\end{array}$ & $\begin{array}{r}693,0 \\
57,2\end{array}$ & $\begin{array}{r}470,0 \\
38,8\end{array}$ & $\begin{array}{r}48,0 \\
4,0\end{array}$ & $\begin{array}{c}1.211 \\
100\end{array}$ & 118 & 1.329 \\
\hline
\end{tabular}

PU: Programa Universitario; AP: Adiestramiento en Práctica: FE: Formación en el Extranjero; Prof. Univ.: Profesor Universitario.

coincidente con la nueva legislación de especialidades evidencia un quiebre en este ritmo ascendente que compromete tanto a los egresados de programas universitarios como los por adiestramiento en práctica y cuya explicación es compleja, sin poder descartar un menor interés por la Pediatría.

La Tabla 2 muestra características demográficas de los postulantes a certificación en cuanto a su procedencia geográfica y género. El origen capitalino ha sido mayoritario, y en el último trienio especialmente marcado; como explicación no puede aducirse una mayor oferta de programas formativos en Santiago.

Una situación distinta sucede con el género de los médicos Pediatras. Hace 23 años la proporción de doctoras era una por cada 1,2 varones. Como lo muestra la Tabla 2 esta proporción fue minimizándose hasta ser igualitaria y, hoy, inversa 0,7 varones por cada doctora.

La Tabla 3 desglosa quinquenalmente el número de postulantes y de médicos que completaron exitosamente el proceso de certificación, lo que al inicio es mayor por la cer-
Tabla 2. Origen y género de los médicos certificados en Pediatría, distribuidos según quinquenios

\begin{tabular}{|ccc|}
\hline Período & Santiago/Provincia* & Hombre/Mujer \\
\hline $1984-1988$ & $1,1 / 1$ & $1,2 / 1$ \\
$1989-1993$ & $2,2 / 1$ & $1,1 / 1$ \\
\hline $1994-1998$ & $2,1 / 1$ & $1,0 / 1$ \\
$1999-2003$ & $2,0 / 1$ & $0,7 / 1$ \\
\hline $2004-2008$ & $2,5 / 1$ & $0,6 / 1$ \\
$2009-2012$ & $2,1 / 1$ & $0,7 / 1$ \\
\hline
\end{tabular}

*Domicilio declarado al postular.

Tabla 3. Postulaciones y certificaciones en Pediatría en el período 1985-2012

\begin{tabular}{|cccc|}
\hline $\begin{array}{c}\text { Período } \\
\text { Años }\end{array}$ & $\begin{array}{c}\text { Postulaciones } \\
\overline{\mathbf{x}} / \mathbf{a n ̃ o}\end{array}$ & $\begin{array}{c}\text { Certificados } \\
\overline{\mathbf{x}} / \mathbf{a n ̃ o}\end{array}$ & $\begin{array}{c}\text { Aprobación } \\
\text { \% }\end{array}$ \\
\hline $1984-1988$ & 136 & 113,4 & 83 \\
\hline $1989-1993$ & 25 & 24,6 & 97 \\
\hline $1994-1998$ & 32 & 23,2 & 73 \\
\hline $1999-2003$ & 47 & 37,0 & 79 \\
$2004-2008$ & 55 & 47,4 & 86 \\
\hline $2009-2012$ & 28 & 25,3 & 90 \\
\hline
\end{tabular}


Tabla 4. Exámenes escritos y prácticos durante el período 2002-2012

\begin{tabular}{|llcccc|}
\hline & & Postulantes & Aprobados & Reprobados & Total \\
Examen escrito & $\mathrm{n}$ & 108 & 101,0 & 28,0 & 129 \\
& $\overline{\mathrm{x}}$ anual & 10,1 & 2,8 & & \\
Examen práctico & $\%$ & 78,3 & 21,7 & & 147 \\
& $\mathrm{n}$ & 100 & 123,0 & 24,0 & \\
& $\overline{\mathrm{x}}$ anual & 12,3 & 2,4 & & \\
\hline
\end{tabular}

tificación de los Profesores Universitarios. El universo de médicos que no consiguió aprobar la certificación $(15,3 \%)$ se explica por causas como no reunir los requisitos de postulación, o, de tenerlos, no aceptar dar examen o fracasar en éste. A partir del año 2002 se incorpora en Pediatría el examen escrito, que a la fecha han rendido 108 postulantes, que con las repeticiones totalizan 129 exámenes. Este ha sido un gran paso en la certificación, cumpliendo un justo rol seleccionador de los candidatos capacitados con 78,3\% de aprobación cumplidos once años de vida del examen. Los candidatos fracasados en el examen tienen otras dos opciones, lo que explica la disparidad entre el número de certificaciones y de aprobaciones. El buen rendimiento del examen escrito se expresa en la menor tasa de rechazo del examen práctico posterior, que alcanza en igual período de once años a $16,3 \%$ (Tabla 4).

\section{¿Cómo tiene diseñado CONACEM el proceso de Recertificación?}

La recertificación impone requisitos generales comunes a todas las especialidades que son: a) Estar certificado como especialista por CONACEM; b) Estar ejerciendo activamente la especialidad; c) Detentar una trayectoria profesional éticamente intachable; d) Completar 100 puntos (créditos), con posterioridad a la última certificación, según la escala confeccionada por CONACEM (Tablas 5 y 6). Este reglamento enfatiza la Educación Continua, al considerarla como único requisito obligatorio con al menos el $40 \%$ de los créditos, y de calidad demostrable, con actividades controladas en asistencia y evaluadas formalmente.

Hay que presentar el currículum vitae de los últimos 10 años y cancelar los aranceles correspondientes.
Los candidatos que no alcancen puntaje o no deseen presentar la documentación requerida, tendrán derecho a solicitar la evaluación teórica y práctica correspondiente.

El proceso de recertificación está por empezar y dada su complejidad y magnitud se requerirá de un esfuerzo mancomunado de todas las instancias de la Medicina Nacional para que lo apoyen y de CONACEM para que lo lleve a afecto.

Mirando la medicina chilena y sus potencialidades, el nivel de desarrollo nacional y los cambios sociológicos y legales ocurridos, el resolver el déficit de especialistas, con diferencias propias entre especialidades, depende en general de tres pilares intrínsecos y uno relacionado. Lo intrínseco dice referencia con: 1) el impulso para perfeccionar y expandir los programas universitarios de formación acreditados o en vías de hacerlo y aumentar sus cupos; 2) el apoyo sostenido de la autoridad sanitaria en cuanto a proveer plazas, remuneraciones, estímulos y equipamiento humano y tecnológico para fomentar la vía de certificación por adiestramiento en práctica y políticas de radicación en las zonas de destinación, lo que ha sido expresado como un objetivo cierto por la autoridad actual; 3) el desarrollo con carácter orgánico, sostenido, controlado, evaluado, y de calidad probada, de programas de

Tabla 5. Origen de los créditos necesarios para la Recertificación en CONACEM (mínimo 100)

\begin{tabular}{ll} 
1. Actividades asistenciales & Máximo $50 \%$ \\
2. Actividades académicas & Máximo $50 \%$ \\
3. Actividades de Educación Continua & Máximo $70 \%$ \\
4. Otras actividades & Mínimo $40 \%$ \\
\hline
\end{tabular}


Tabla 6. Reglamento de Recertificación. Créditos según actividades

\begin{tabular}{|c|c|c|}
\hline \multicolumn{3}{|l|}{ 1. Actividades Asistenciales (máx. 50 puntos) } \\
\hline \multicolumn{2}{|c|}{ Trabajo con contrato o convenio en departamento o servicio asistencial clínico, público o privado } & $5 /$ año \\
\hline \multicolumn{2}{|c|}{ Trabajo en consulta privada solamente (a evaluar por comité correspondiente) } & 3 / año \\
\hline \multicolumn{3}{|l|}{ 2. Actividades Académicas (máx. 50 puntos) } \\
\hline Docente Universitario & $\begin{array}{l}\text { Profesor (cualquier categoría) } \\
\text { Instructor o Ayudante } \\
\text { Con asignación de docencia }\end{array}$ & $\begin{array}{l}10 \text { / año } \\
5 / \text { año } \\
2 \text { / año }\end{array}$ \\
\hline $\begin{array}{l}\text { Publicaciones en revistas con comité editorial, nacionales o } \\
\text { extranjeras }\end{array}$ & $\begin{array}{l}\text { Autor principal o líder del grupo } \\
\text { Coautor }\end{array}$ & $\begin{array}{r}10 \mathrm{c} / \mathrm{u} \\
5 \mathrm{c} / \mathrm{u}\end{array}$ \\
\hline Capítulos de libros & $\begin{array}{l}\text { Autor } \\
\text { Coautor }\end{array}$ & $\begin{array}{r}10 \mathrm{c} / \mathrm{u} \\
3 \mathrm{c} / \mathrm{u}\end{array}$ \\
\hline \multicolumn{2}{|l|}{ Director o Docente en curso de perfeccionamiento } & 5 por curso \\
\hline \multicolumn{2}{|l|}{ Conferencista o Integrante de Mesa Redonda en Congreso } & 5 por congreso \\
\hline \multicolumn{3}{|c|}{ 3. Actividades de Perfeccionamiento o de Educación Continua (máx. 70 / mín. 40) } \\
\hline \multicolumn{2}{|l|}{ Becas y cursos de perfeccionamiento } & 10 por mes (máximo 30) \\
\hline \multicolumn{2}{|l|}{$\begin{array}{l}\text { Asistencia certificada a cursos y congresos de la especialidad, } \\
\text { nacionales y extranjeros }\end{array}$} & $3 \mathrm{c} / \mathrm{u}$ \\
\hline $\begin{array}{l}\text { Asistencia certificada a cursos de perfeccionamiento con más de } \\
10 \text { h lectivas }\end{array}$ & $\begin{array}{l}\text { Con evaluación } \\
\text { Sin evaluación }\end{array}$ & $\begin{array}{l}6 \mathrm{c} / \mathrm{u} \\
3 \mathrm{c} / \mathrm{u}\end{array}$ \\
\hline $\begin{array}{l}\text { Presentación trabajos de la especialidad a congresos nacionales o } \\
\text { extranjeros }\end{array}$ & $\begin{array}{l}\text { Autor principal o líder de grupo } \\
\text { Coautor }\end{array}$ & $\begin{array}{l}3 \mathrm{c} / \mathrm{u} \\
2 \mathrm{c} / \mathrm{u}\end{array}$ \\
\hline \multicolumn{2}{|l|}{ Asistencia certificada a reuniones clínicas } & $\begin{array}{l}3 \text { por año } \\
(0,2 \text { puntos por reunión) }\end{array}$ \\
\hline \multicolumn{3}{|l|}{ 4. Otras Actividades (máx. 40 puntos) } \\
\hline \multicolumn{2}{|c|}{ Jefatura de Servicio público por concurso o de Departamento universitario } & 10 por año \\
\hline \multicolumn{2}{|c|}{ Miembro del directorio o de comité científico de la sociedad científica pertinente } & 5 por una vez \\
\hline \multicolumn{2}{|l|}{ Miembro de comité editorial de publicación de la especialidad } & 5 por comité \\
\hline \multicolumn{2}{|l|}{ Miembro de sociedad científica de la especialidad } & $\begin{array}{l}5 \text { por sociedad } \\
\text { (máximo 10) }\end{array}$ \\
\hline \multicolumn{2}{|l|}{ Otras (a evaluar) } & 10 máximo \\
\hline
\end{tabular}

educación médica continua, presencial o a distancia. La obsolescencia progresiva de la competencia profesional es un síndrome universal con muchas formas subclínicas que pasan desapercibidas, y que en general hay reticencia a reconocerlas. La Educación Continua es, hasta ahora, el único tratamiento conocido para la obsolescencia del saber y del actuar.

El pilar restante, no ligado a las especialidades, pero con notable repercusión sobre ellas, es la calidad y cobertura de la medicina de atención primaria. Si ella es deficitaria, por número, eficiencia o ambas razones, la demanda de especialistas será enorme y la capacidad disponible de ellos sobrepasada. No puede haber desarrollo de especialidades sin el propio de la atención primaria.

\section{¿Qué trae el futuro inmediato a nuestra medicina chilena?}

Varios son los hechos que se vislumbran o ya están en escena demandando solución. Señalaré: 1) la creciente demanda de especialistas, ahora acentuada por los imperativos legales que requieren ese concurso; 2) Acortamiento de los programas de especialidades derivadas, con nuevas vías de ingreso a ellas, 3) Fortalecimiento de la vía de adiestramiento 
en práctica. El centro de gravedad de esta vía debería pasar desde el notable esfuerzo individual de cada candidato, a una condición de mayor ayuda y respaldo del sistema con organización nacional; 4) Trabajo cooperativo entre universidades para el desarrollo de programas complejos; 5) Crecientes solicitudes de certificación por médicos, chilenos o no, formados en el extranjero; 6) Aparición de nuevas especialidades o subespecialidades,

Cualquier solución al acuciante problema de la carencia de especialistas requiere del concurso de todos los actores de la medicina chilena. Relegar esta responsabilidad sólo a la autoridad sanitaria ha probado conseguir soluciones tardías y parciales e insatisfacciones crecientes.

Además del fortalecimiento de la atención primaria, una propuesta de solución al déficit de especialistas requiere: 1) Llevar al máximo el número de programas universitarios acreditados, y sus cupos, manteniendo la calidad. Aunque lo anterior abre una solución sólo a mediano plazo, no hay razón para no empezar. Esta generación de especialistas debe constituir el factor multiplicador cuando asentados a lo largo del país, idealmente en parejas, con destinación geográfica conocida desde el inicio de su formación, puedan sustentar la formación por adiestramiento en práctica de otros médicos en sus lugares de destino; b) La autoridad sanitaria debería estimular estas desti- naciones con plazas de trabajo, facilidades de asentamiento, dotación de personal de apoyo, equipamiento tecnológico y remuneraciones atractivas. Se iniciaría un círculo virtuoso interesando a profesionales a usar la vía de formación en práctica y rompería la limitante actual para que esos interesados no tengan que interrumpir por dos o tres años su vida familiar e intereses personales para radicarse lejos de su residencia en centros universitarios de formación; realista es que esa formación se haga trabajando en su ciudad de residencia, bajo la supervisión de dos especialistas acreditados y programas predeterminados.

Los candidatos para la vía de adiestramiento en práctica requieren el soporte de una educación continua eficaz: En este campo las Facultades de Medicina, el Colegio Médico de Chile A.G. y las Sociedades Científicas pueden contribuir substantivamente. En suma, una solución integral para la carencia de especialistas requiere el concurso de todas las potencialidades de la medicina chilena. Ellas existen y necesitan ser coordinadas y orientadas. No hacerlo merece más que una simple explicación.

Potenciales conflictos de interés: Este trabajo cumple con los requisitos sobre consentimiento/asentimiento informado, comité de ética, financiamiento, estudios animales y sobre la ausencia de conflictos de intereses según corresponda. 